Portland State University

PDXScholar

Mathematics and Statistics Faculty

Fariborz Maseeh Department of Mathematics

Publications and Presentations

and Statistics

2005

\title{
Stable Motions of Vehicle Formations
}

Anca Williams

Portland State University

Gerardo Lafferriere

Portland State University, Gerardol@pdx.edu

J. J. P. Veerman

Portland State University, veerman@pdx.edu

Follow this and additional works at: https://pdxscholar.library.pdx.edu/mth_fac

Part of the Control Theory Commons, and the Non-linear Dynamics Commons

Let us know how access to this document benefits you.

\section{Citation Details}

Williams, Anca; Lafferriere, Gerardo; and Veerman, J. J. P., "Stable Motions of Vehicle Formations" (2005). Mathematics and Statistics Faculty Publications and Presentations. 132.

https://pdxscholar.library.pdx.edu/mth_fac/132

This Post-Print is brought to you for free and open access. It has been accepted for inclusion in Mathematics and Statistics Faculty Publications and Presentations by an authorized administrator of PDXScholar. Please contact us if we can make this document more accessible: pdxscholar@pdx.edu. 


\title{
Stable motions of vehicle formations
}

\author{
A. Williams, G. Lafferriere, J.J.P. Veerman
}

\begin{abstract}
We investigate stable maneuvers for a group of autonomous vehicles while moving in formation. The allowed decentralized feedback laws are factored through the Laplacian matrix of the communication graph. We show that such laws allow for stable circular or elliptical motions for certain vehicle dynamics. We find necessary and sufficient conditions on the feedback gains and the dynamic parameters for convergence to formation. In particular, we prove that for undirected graphs there exist feedback gains that stabilize rotational (or elliptical) motions of arbitrary radius (or eccentricity). In the directed graph case we provide necessary and sufficient conditions on the curvature that guarantee stability for a given choice of feedback gains. We also investigate stable motions involving reorientation of the formation along the direction of motion.

Keywords: formation stability, formation maneuvers, graph Laplacian, decentralized control.
\end{abstract}

\section{INTRODUCTION}

The study of decentralized control of multiple autonomous vehicles offers many interesting challenges. There is a growing literature devoted to such problems [1], [2], [3], [4], [5], [6], [7], [8], [9], [10], [11], [12], [13], [14], [15]. One of the main goals is to achieve a coordinated objective while using only relative information concerning positions and velocity. More precisely, the goal of the vehicles is to achieve and maintain pre-specified relative positions and orientations with respect to each other while performing joint maneuvers. Each vehicle is provided information only from a subset of the group. The specific subset is given through the set of "neighbors" in a communication digraph. This digraph remains fixed during the motions and need not be related in any way to the actual position of the vehicles. In this paper we study the stability of circular and elliptical motions while maintaining an arbitrary formation. We also sharpen the estimates in [16] that relate the feedback gains to the curvature of the motion and investigate the problem of reorienting the formation while moving.

The feedback scheme investigated here was proposed by Fax and Murray in [2]. We point out that the issues investigated here are related to the problem of consensus seeking by autonomous agents ([17]). For additional background we refer the reader to [6] and the references therein.

The paper is organized as follows. In Section II we set up the basic model. The relevant graph theoretic facts are collected in Section III. The main results are proved in Section IV. Various motions of the formation are illustrated in Section V and VI.

All three authors are with the Department of Mathematics and Statistics, Portland State University, Portland, OR 97207-0751, USA. Email: ancaw@pdx.edu, gerardoL@pdx.edu, veerman@pdx.edu

G. Lafferriere was supported in part by NSF grant DMS-0408334 and by a Career Support grant from Portland State University.

\section{MOdel}

We assume we are given $N$ vehicles with the same dynamics

$$
\dot{x}_{i}=A_{v e h} x_{i}+B_{v e h} u_{i} \quad i=1 \ldots N \quad x_{i} \in \mathbb{R}^{2 n}
$$

where the entries of $x_{i}$ represent $n$ configuration variables for vehicle $i$ and their derivatives, and the $u_{i}$ represent control inputs. We will be specially interested in the case where the vehicles move in two dimensions and the matrices $A_{v e h}$ and $B_{v e h}$ are of the form

$$
A_{\text {veh }}=\left(\begin{array}{cccc}
0 & 1 & 0 & 0 \\
0 & a_{22} & 0 & a_{24} \\
0 & 0 & 0 & 1 \\
0 & a_{42} & 0 & a_{44}
\end{array}\right) \quad B_{\text {veh }}=\left(\begin{array}{ll}
0 & 0 \\
1 & 0 \\
0 & 0 \\
0 & 1
\end{array}\right) .
$$

The form of the first and third rows of $A_{v e h}$ and $B_{v e h}$ is determined by the fact that the even-numbered coordinates represent the velocities of the (previous) odd-numbered coordinates and that the controls are the accelerations (this even holds for $n>2$ ). The zeros in the even columns of $A_{v e h}$ are necessary if we want the vehicles to converge to formation (see [5] Proposition 3.1 and [16] Proposition 4.2). We will explore below the effect of the other entries.

We will refer to the odd-numbered entries of $x=$ $\left(x_{1}, \ldots, x_{N}\right)^{T}$ as position-like variables and to the evennumbered entries as velocity-like variables. We will use the notation $x_{p}=\left(\left(x_{p}\right)_{1}, \ldots,\left(x_{p}\right)_{N}\right)^{T}, x_{v}=$ $\left(\left(x_{v}\right)_{1}, \ldots,\left(x_{v}\right)_{N}\right)^{T}$ to denote the vectors of position-like and velocity-like variables, so $x=x_{p} \otimes\left(\begin{array}{l}1 \\ 0\end{array}\right)+x_{v} \otimes\left(\begin{array}{l}0 \\ 1\end{array}\right)$ (where $\otimes$ denotes the Kronecker product).

Definition 2.1: A formation is a vector $h=h_{p} \otimes\left(\begin{array}{l}1 \\ 0\end{array}\right) \in$ $\mathbb{R}^{2 n N}$ (where $\otimes$ denotes the Kronecker product). The $N$ vehicles are in formation $h$ at time $t$ if there are vectors $q, w \in \mathbb{R}^{n}$ such that $\left(x_{p}\right)_{i}(t)-\left(h_{p}\right)_{i}=q$ and $\left(x_{v}\right)_{i}(t)=$ $w$, for $i=1 \ldots N$. The vehicles converge to formation $h$ if there exist $\mathbb{R}^{n}$-valued functions $q(\cdot), w(\cdot)$ such that $\left(x_{p}\right)_{i}(t)-\left(h_{p}\right)_{i}-q(t) \rightarrow 0$ and $\left(x_{v}\right)_{i}(t)-w(t) \rightarrow 0$, as $t \rightarrow \infty$, for $i=1 \ldots N$ (where $x_{p}$ and $x_{v}$ are as indicated above).

Figure 1 illustrates the interpretation of the vectors in the definition.

For more convenient mathematical treatment we introduce the following space associated with each formation vector.

Definition 2.2: Let $h=h_{p} \otimes\left(\begin{array}{l}1 \\ 0\end{array}\right) \in \mathbb{R}^{2 n N}$, let $\mathbf{1}_{N}$ denote the all ones vector of size $N$, and let $\mathbf{e}_{j}, j=1, \ldots, 2 n$ 


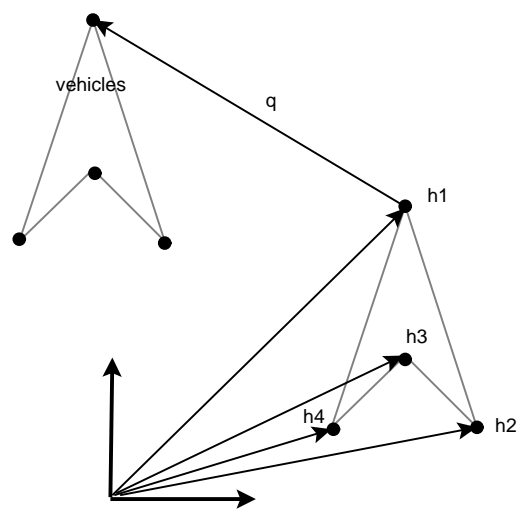

Fig. 1. Four vehicles in arrow tip formation

denote the standard basis vectors in $\mathbb{R}^{2 n}$. Set $W=\left\{\mathbf{1}_{N} \otimes\right.$ $\left.\mathbf{e}_{j}: j=1, \ldots, 2 n\right\}$. We define the $h$-formation space $\mathcal{F}_{h}$ by

$$
\mathcal{F}_{h}=h+\operatorname{span} W=\left\{x \mid \exists \gamma \in \mathbb{R}^{2 n}: x-h=\mathbf{1}_{N} \otimes \gamma\right\}
$$

Notice that $x-h=\mathbf{1}_{N} \otimes \gamma$ is equivalent to $\left(x_{p}\right)_{i}-\left(h_{p}\right)_{i}=q$ and $\left(x_{v}\right)_{i}=w$ where $\gamma=q \otimes\left(\begin{array}{l}1 \\ 0\end{array}\right)+w \otimes\left(\begin{array}{l}0 \\ 1\end{array}\right)$.

With this definition $x$ is in formation $h$ if and only if $x \in \mathcal{F}_{h}$ and $x(t)$ converges to formation $h$ if and only if the distance from $x(t)$ to the space $\mathcal{F}_{h}$ tends to zero as $t \rightarrow \infty$.

To complete the model we are also given a digraph $\Gamma$ which captures the communication links between vehicles (see Section III). Each vertex represents a vehicle and there is a directed edge from one vertex to another if there is a communication link sending information from the first vehicle to the second. The second vehicle uses this information in a feedback formula to adjust its own state. We say that the first vehicle is a neighbor of the second. For each vehicle $i$, $\mathbb{J}_{i}$ denotes the set of its neighbors. The decentralized nature of the control is encoded into the fact that controls $u_{i}$ are functions of $x_{j}-x_{i}$ and $h_{j}-h_{i}$ for each $j \in \mathbb{J}_{i}$. Note, however, that this still requires all vehicles to agree on a global reference frame with respect to which the differences $x_{j}-x_{i}$ can be described.

A natural way to combine the relative information (as done in [2]) is to define output functions $y_{i}$ computed from an average of the relative displacements (and velocities) of the neighboring vehicles as follows

$$
y_{i}=\left(x_{i}-h_{i}\right)-\frac{1}{\left|\mathbb{J}_{i}\right|} \sum_{j \in \mathbb{J}_{i}}\left(x_{j}-h_{j}\right) \quad i=1, \ldots, N .
$$

where $\left|\mathbb{J}_{i}\right|$ indicates the number of neighbors to vehicle $i$. We will make a slight modification here to allow for the possibility that a vehicle might receive no information and so the corresponding vertex in the graph would have no neighbors. This situation occurs naturally if one of the vehicles is a designated leader around whose motions the others should adjust theirs. We will define the output functions $z_{i}$ by

$$
z_{i}= \begin{cases}\frac{1}{\left|\mathbb{J}_{i}\right|} \sum_{j \in \mathbb{J}_{i}}\left(\left(x_{i}-h_{i}\right)-\left(x_{j}-h_{j}\right)\right) & \text { if }\left|\mathbb{J}_{i}\right| \neq 0 \\ 0 & \text { otherwise }\end{cases}
$$

for $i=1, \ldots, N$. As a result, the corresponding output vector $z$ can be written as $z=L(x-h)$ where $L=$ $L_{\Gamma} \otimes I_{2 n}$ and $L_{\Gamma}$ is the (directed) Laplacian matrix of the communication graph $\Gamma$ (see Section III).

Collecting the equations for all the vehicles into a single system we obtain

$$
\begin{aligned}
\dot{x} & =A x+B u \\
z & =L(x-h)
\end{aligned}
$$

with $A=I_{N} \otimes A_{v e h}, B=I_{N} \otimes B_{v e h}$.

We are interested in studying the existence of feedback matrices $F$ such that the solutions to

$$
\dot{x}=A x+B F L(x-h)
$$

converge to formation $h$. This is a stabilization problem involving output feedback. In order to take advantage of the block structure of the matrices $A, B$, and $L$ we will restrict our study to matrices $F$ of the form $F=I_{N} \otimes F_{v e h}$ (a particular "decentralized" control law where the same feedback law applies to all vehicles). In this case, we can write Equation (3) as follows

$$
\dot{x}=I_{N} \otimes A_{v e h} x+L_{G} \otimes B_{v e h} F_{v e h}(x-h)
$$

The main purpose of the paper is to investigate the relationship between of feedback matrices $F_{v e h}$ that guarantee convergence to formation and the structure of $A_{v e h}$.

\section{Algebraic Graph TheORY}

Here we include only the minimal terminology from graph theory to treat the problem at hand (for standard terms see [18]). A directed graph or digraph $\Gamma$ consists of a finite set $\mathcal{V}$ of vertices and a set $\mathcal{E} \subseteq V \times V$ (the directed edges). We will assume that the digraph has no loops, that is $(x, y) \in \mathcal{E}$ implies $x \neq y$. If the digraph has the property that $(x, y) \in \mathcal{E}$ implies $(y, x) \in \mathcal{E}$ we will say that it is undirected. In the vehicle context this corresponds to having bidirectional communication.

A (directed) walk in a digraph is a finite sequence of edges $\left(a_{k}, b_{k}\right) k=1, \ldots, r$ such that $b_{k}=a_{k+1}$ for $k=1, \ldots, r-$ 1. A walk with distinct vertices is called a (directed) path.

Let $\Gamma$ denote a digraph with vertex set $\mathcal{V}=\{i: i=$ $1, \ldots, N\}$ and edge set $\mathcal{E}$. Let $\mathrm{Mat}_{N}$ denote the set of all square $N \times N$ matrices with real entries. The adjacency matrix of $\Gamma$ is the matrix $Q \in$ Mat $_{N}$ with entries

$$
q_{i j}=\left\{\begin{array}{ll}
1 & \text { if }(j, i) \in \mathcal{E}, \\
0 & \text { otherwise }
\end{array} \quad(i, j \in \mathcal{V}) .\right.
$$

When $\Gamma$ is undirected, the matrix $Q$ is symmetric. The indegree matrix of $\Gamma$ is the diagonal matrix $D \in \operatorname{Mat}_{\mathcal{V}}(\mathbb{R})$ with diagonal entries

$$
d_{i i}=|\{j \in \mathcal{V}:(j, i) \in \mathcal{E}\}| \quad(i \in \mathcal{V}) .
$$

The directed Laplacian of $\Gamma$ is the matrix defined by ([16])

$$
L_{\Gamma}=D^{+}(D-Q),
$$


where $D^{+}$is the (Moore-Penrose) pseudoinverse of $D$. If $D$ is invertible then $L_{\Gamma}=I_{N}-D^{-1} Q$ (see [19], [1] for general properties in this case). We use the word directed to distinguish this matrix from the most common definition of Laplacian in the graph theory literature ([18]) as $M_{\Gamma} M_{\Gamma}^{T}$ where $M_{\Gamma}$ is the incidence matrix of $\Gamma$ (but see also [20]). This definition always results in the same symmetric matrix regardless of whether the graph is directed or not. We prefer the first definition since it is the matrix $L_{\Gamma}$ which is the most relevant to this problem. Both definitions agree for undirected graphs.

The eigenvalues of the Laplacian have several well known properties. If the graph is undirected then the eigenvalues of $L_{\Gamma}$ are real. A key property of $L_{\Gamma}$ that we will use is that zero is an eigenvalue of $L_{\Gamma}$ and the all ones vector $\mathbf{1}$ is an associated eigenvector (but in general there could be others). In all cases, all the eigenvalues of $L_{\Gamma}$ lie in the circle of radius 1 centered at the point $1+0 i$ in the complex plane. In particular, except for zero, all eigenvalues have positive real part (for additional properties see [6]).

Definition 3.1: A rooted directed tree is a digraph $T$ with the following properties:

- $T$ has no cycles.

- There exists a vertex $v$ (the root) such that there is a (directed) path from $v$ to every other vertex in $T$.

The following result was proved in [6].

Proposition 3.2: Let $G$ denote a (loopless) digraph. Then, zero is an eigenvalue of algebraic multiplicity one for the directed Laplacian $L_{\Gamma}$ if and only if $\Gamma$ has a rooted directed spanning tree.

In fact, this is a consequence of a more general result on the structure of the null space of the Laplacian (see [16]). For additional graph theoretic terms and results see [21]. In what follows we will only be interested in the case when zero is an eigenvalue of multiplicity one of $\Gamma$.

\section{Stabilizability}

We are interested in stabilizing gains for matrices $A_{v e h}$ and $B_{v e h}$ given by (2). First we recall the general stabilization result.

Theorem 4.1: ([6]) Let $\Gamma$ be a digraph with the property that zero is an eigenvalue of the directed Laplacian $L_{\Gamma}$ of algebraic multiplicity one. Then the matrix $A_{v e h}+\lambda B_{v e h} F_{v e h}$ is stable (Hurwitz) for each nonzero eigenvalue $\lambda$ of $L_{\Gamma}$ if and only if, for every $h$, every solution of (4) converges to formation $h$.

We now investigate the existence of $F_{v e h}$ that make $A_{v e h}+\lambda B_{v e h} F_{v e h}$ stable for the matrices of the form (2) (in particular, the vehicles are moving on a plane). Towards this end we introduce the following submatrix of $A_{v e h}$

$$
A_{\text {for }}=\left(\begin{array}{ll}
a_{22} & a_{24} \\
a_{42} & a_{44}
\end{array}\right) .
$$

We will search for feedback which is of the even simpler form

$$
F_{v e h}=\left(\begin{array}{cccc}
f_{1} & f_{2} & 0 & 0 \\
0 & 0 & f_{1} & f_{2}
\end{array}\right)
$$

Necessary and sufficient conditions for $f_{1}, f_{2}$ to stabilize $A_{v e h}+\lambda B_{v e h} F_{v e h}$ where given in [6] for the case when $A_{f o r}$ is diagonal. Here we continue the analysis in [16] for the case when $A_{\text {for }}$ has off-diagonal terms. We consider first the case when $A_{\text {for }}$ has the form

$$
A_{\text {for }}=\left(\begin{array}{cc}
0 & -k \\
k & 0
\end{array}\right)
$$

for some $k>0$. We should think of $A_{f o r}$ as capturing the formation dynamics.

Let $q(x)=x^{2}+s x+p$, be a polynomial where $s=s_{1}+s_{2} i$ and $p=p_{1}+p_{2} i$ are complex numbers. If $\mu$ is a root of $q(x)$ then $\bar{\mu}$ is a root of $r(x)=x^{2}+\bar{s} x+\bar{p}$. Therefore $q(x)$ is stable if and only if $h(x)=q(x) r(x)$ is. Applying Routh's criterion to $h(x)$ (which has real coefficients) results in the following necessary and sufficient conditions for stability of $q(x)$ :

$$
\begin{gathered}
s_{1}>0 \quad 2 p_{1}+s_{1}^{2}+s_{2}^{2}>0 \quad s_{1} p_{1}+s_{2} p_{2}>0 \\
p_{1} s_{1}^{2}+p_{2} s_{1} s_{2}-p_{2}^{2}>0
\end{gathered}
$$

The eigenvalues of $A_{v e h}+\lambda B_{v e h} F_{v e h}$ are the roots of the polynomial $q(x)=\left(x^{2}-\lambda f_{2} x+\left(-\lambda f_{1}\right)\right)^{2}+k^{2} x^{2}$. We can factor $q(x)$ as $q_{+}(x) q_{-}(x)$ where

$$
q_{ \pm}(x)=x^{2}-\lambda f_{2} x+\left(-\lambda f_{1}\right) \pm i x k .
$$

Since the only difference in the factors is in the term $\pm i k x$ we will only show the calculations for $q_{+}(x)$. Writing $\lambda=$ $\alpha+\beta i$ we can apply the above conditions with $s_{1}=-f_{2} \alpha$, $s_{2}=k-f_{2} \beta, p_{1}=-f_{1} \alpha$, and $p_{2}=-f_{1} \beta$. The above formulas become:

$$
\begin{aligned}
& 0<-\alpha f_{2} \\
& 0<-2 \alpha f_{1}+\left(\alpha f_{2}\right)^{2}+\left(k-\beta f_{2}\right)^{2} \\
& 0<\left(-f_{2} \alpha\right)\left(-f_{1} \alpha\right)+\left(k-f_{2} \beta\right)\left(-f_{1} \beta\right) \\
& 0<\left(-f_{1} \alpha\right)\left(f_{2} \alpha\right)^{2}-f_{1} \beta\left(-f_{2} \alpha\right)\left(k-f_{2} \beta\right)-f_{1}^{2} \beta^{2}
\end{aligned}
$$

As mentioned in Section III, we always have $\alpha>0$, so the first inequality is always equivalent to $f_{2}<0$. In the case when $\lambda$ is real $(\beta=0)$, for example when the communication graph is undirected, the last inequality is equivalent to $f_{1}<$ 0 . In this case the other two inequalities are automatically satisfied. We conclude that for real $\lambda$ the inequalities simplify to

$$
f_{1}<0 \quad f_{2}<0
$$

regardless of $k$.

We have now shown:

Proposition 4.2: When the eigenvalues of the Laplacian are real and the vehicle dynamics are given by (5) the vehicles converge to formation if and only if $f_{1}<0$ and $f_{2}<0$.

The sufficiency was also derived in [16] in a different way.

Remark 4.3: It is worth pointing out that in the case of real eigenvalues the same gains achieve stability for any value of $k$ (and hence for arbitrary curvatures). The story 
is quite different in the case of complex eigenvalues as we now see.

Assume now that there are some complex eigenvalues, so $\beta \neq 0$. We still get $f_{2}<0$. The third inequality reduces to $f_{1} \beta k<f_{1} f_{2}\left(\alpha^{2}+\beta^{2}\right)$. Since the Laplacian has real entries, its eigenvalues come in complex pairs. Therefore this last inequality must hold for $\beta$ positive and negative. This implies that the right side must be positive and so again we get $f_{1}<0$. We therefore get the inequality

$$
|k|<-\frac{f_{2}\left(\alpha^{2}+\beta^{2}\right)}{|\beta|},
$$

for those eigenvalues of $L_{\Gamma}$ for which $\beta \neq 0$ (for the others the third inequality above is automatically satisfied). Notice that the absolute value also accounts for the corresponding inequality for $q_{-}(x)$. Since $f_{1}<0$ the second inequality is automatically satisfied. The fourth inequality simplifies to

$$
\beta k>\frac{-f_{1} \beta^{2}-\alpha f_{2}^{2}\left(\alpha^{2}+\beta^{2}\right)}{-f_{2} \alpha} .
$$

Again this must hold for $\beta$ both positive and negative. This requires that the right hand side be negative. This condition is equivalent to

$$
\frac{-f_{1}}{f_{2}^{2}}<\frac{\alpha}{\beta^{2}}\left(\alpha^{2}+\beta^{2}\right)
$$

Under this condition the fourth inequality then reduces to

$$
|k|<\frac{-f_{1}|\beta|}{f_{2} \alpha}-\frac{f_{2}\left(\alpha^{2}+\beta^{2}\right)}{|\beta|}
$$

Since $f_{1}$ and $f_{2}$ are negative the inequality (8) implies (6). So the conditions we obtain for $k$ are just given by (7) and (8). We have shown the following.

Proposition 4.4: Necessary and sufficient conditions for system (5) to converge to formation are $f_{1}<0, f_{2}<0$ and that $k$ satisfy (7) and (8) for all eigenvalues of the Laplacian with non-zero imaginary part.

These conditions are sharper than those in [16] as can be seen by choosing $f_{1}=-1, f_{2}=-1$ and $k=0.05$ when the graph is the directed cycle on 5 vertices, for example. In this case both (7) and (8) are satisfied but the conditions in [16] fail.

We now analyze the paths that are followed while maintaining the formation. Assume that the vehicles start in formation. That is, if $x_{0}$ is the initial condition, then $x_{0}=$ $h+\mathbf{1}_{N} \otimes \gamma_{0}$. Let $x(t)$ denote the solution to the system

$$
\begin{aligned}
\dot{x} & =\left(I_{N} \otimes A_{v e h}\right) x \\
x(0) & =x_{0} .
\end{aligned}
$$

We want to show that there exists a function $\gamma(t)$ such that $x(t)=h+\mathbf{1}_{N} \otimes \gamma(t)$ for all $t$ (see Definition 2.2). Notice that, in this case, $x(t)$ is also a solution of System 4, and so solutions of (9) represent the trajectories of the vehicles while in formation. The solution to (9) has the form $x(t)=$
$I_{n} \otimes e^{A_{v e h} t} x_{0}$. Then, by expanding the exponential into its power series, we get

$$
\begin{aligned}
x(t)= & I_{N} \otimes e^{A_{v e h} t} h+\left(I_{N} \otimes e^{A_{v e h} t}\right)\left(\mathbf{1}_{N} \otimes \gamma_{0}\right) \\
= & \left(I_{N} \otimes I_{2 n}\right) h+\left(I_{N} \otimes \sum_{j>0} \frac{A_{v e h}^{j} t^{j}}{j !}\right) h \\
& +\mathbf{1}_{N} \otimes\left(e^{A_{v e h} t} \gamma_{0}\right) \\
= & h+\mathbf{1}_{N} \otimes\left(e^{A_{v e h} t} \gamma_{0}\right)
\end{aligned}
$$

where the second term vanishes because the even-numbered entries of $h$ are zero and the odd-numbered columns of $A_{v e h}$ are zero. This is as desired with $\gamma(t)=e^{A_{v e h} t} \gamma_{0}$. For the system (9) the functions $x_{2 i}^{2}+x_{4 i}^{2}$ are integrals of motion for $i=1, \ldots, N$ (we are in the case $n=2$ ). We have shown the following.

Proposition 4.5: If the vehicles start in formation with initial velocity $v_{0}$ then the vehicles maintain the formation while moving along a circular path of radius $v_{0} / k$ at constant speed $v_{0}$. If $v_{0}=0$ then the vehicles will approach formation and a fixed point location in space.

If the vehicles do not start in formation then, under the assumptions of either Proposition 4.2 or Proposition 4.4, the vehicles will converge to formation while asymptotically approaching such a circular path where $v_{0}$ depends on the initial velocities. Finally we point out that if in the matrix $A_{\text {for }}$ we set $a_{22}=a_{44}=0, a_{24}=-k^{2}$ and $a_{42}=1$, then the exact same inequalities for $k$ guarantee convergence to formation and this time the functions $x_{2 i}^{2}+k^{2} x_{4 i}^{2}$ are integrals of motion for $i=1, \ldots, N$. Therefore the in-formation trajectories are ellipses (of eccentricity $\sqrt{1-k^{4}}$ if $k<1$ ).

\section{EXAMPLES}

We illustrate the main results with several numerical examples. In all these figures, there are six vehicles and the desired formation is an arrow tip pointing to the right. In some of the plots involving circles of small radius the formation was scaled up in order to show details of the trajectories (the size of the formation does not affect convergence). All vehicles start lined up in a row with some initial velocity. Figure 2 shows the trajectories when the communication graph is a directed cycle in six vertices and $v_{0} \approx 30.15$. This results in circles of radius of about 150 and curvature 0.007 . In Figure 3 the same graph is used but $k$ is larger $(k=5)$ and $v_{0} \approx 1$, which results in a radius of approximately 0.2 (the formation was scaled up). In this case the larger $k$ required different gains to guarantee convergence. Figure 4 has the same graph and parameters as Figures 2 but the value of $k$ is increased to the point that Inequality (8) fails and so the vehicles do not converge to formation.

Figure 5 illustrates the effect of using different graphs, but the same feedback gains. Compare Fig 5 where $\Gamma$ is the undirected 6-path to Fig. 3 where $\Gamma$ is the directed 6-cycle. In Fig. 5 the vehicles take a much longer time to achieve formation.

Figure 6 illustrates the effect of a change of gains. The solid and dashed formation lines indicate the position of the 


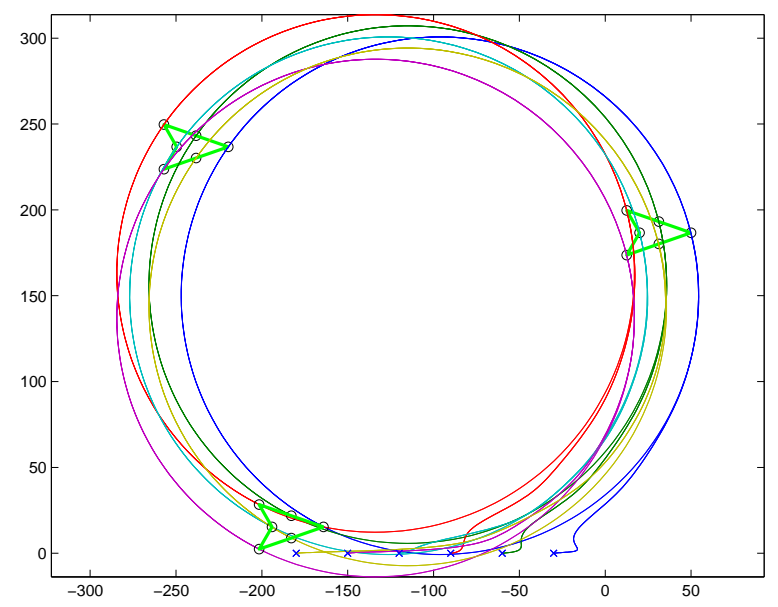

Fig. 2. Rotation with $k=0.2, f_{1}=-2, f_{2}=-3$

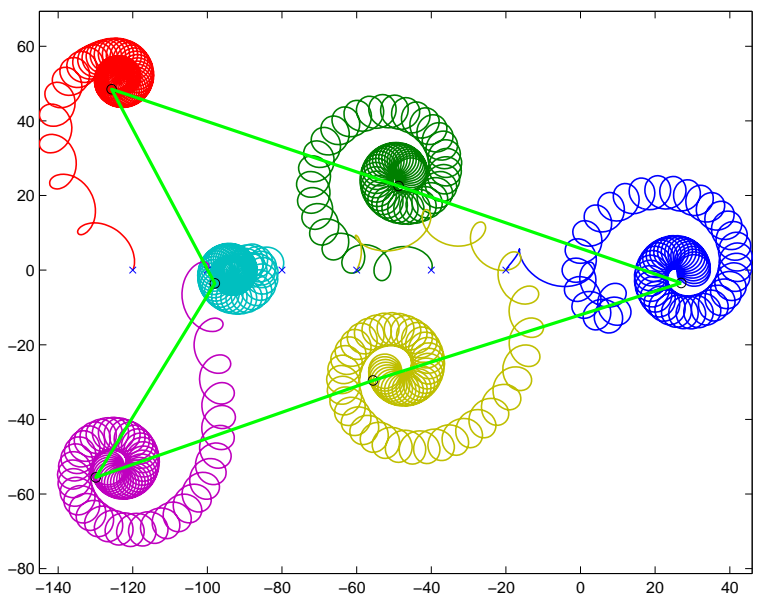

Fig. 3. Rotation with $k=5, f_{1}=-4, f_{2}=-6$.

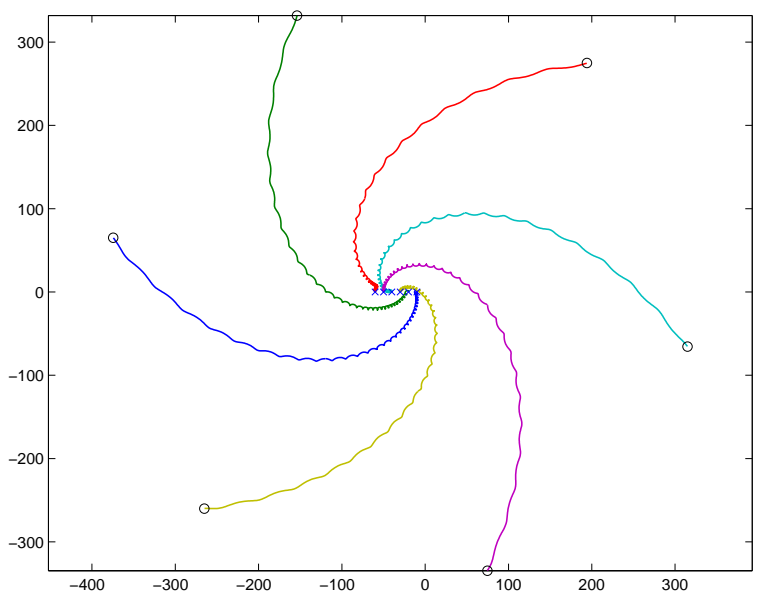

Fig. 4. Rotation with $k=10, f_{1}=-2, f_{2}=-3$. Inequality (8) fails. Vehicles do not converge to formation.

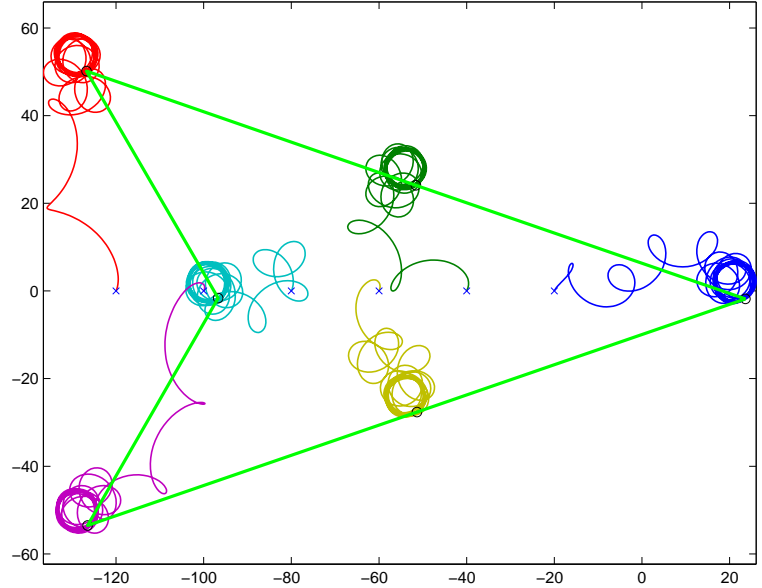

Fig. 5. Rotation with $k=5, f_{1}=-4, f_{2}=-6 . \Gamma$ is the undirected 6-path.

vehicles at the end of two runs of the same duration but with different gains. The vehicles connected by the dashed lines are visibly further away from formation than the ones connected by the solid lines.

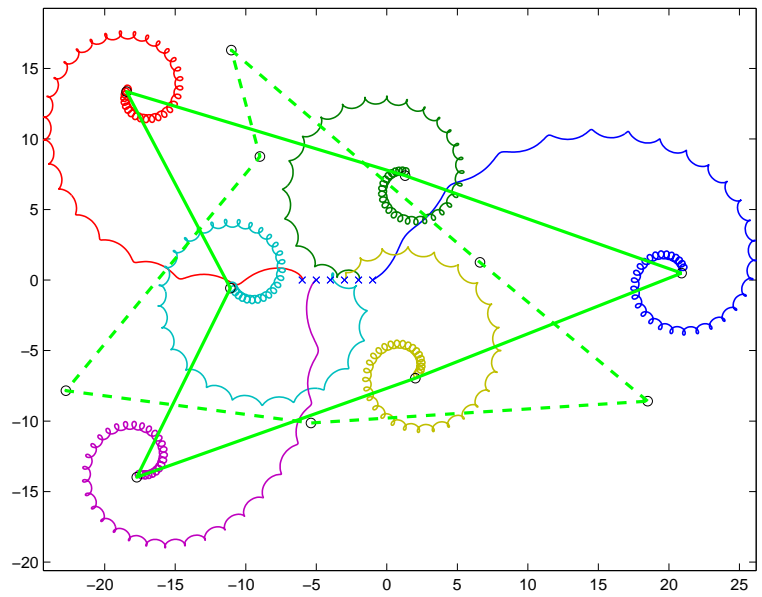

Fig. 6. $\quad \Gamma$ is undirected cycle, $k=5$. Gains are $f_{1}=-2, f_{2}=-3$ (solid) and $f_{1}=-1, f_{2}=-1$ (dashed).

In the case of zero initial velocity the vehicles still converge to formation but they do not rotate around a circle (actually the radius is zero). Figure 7 illustrates two runs with initial zero velocity and two different values of $k(k=1$ in bold, $k=10$ in normal). The larger $k$ produces the longer excursions.

Finally, Figures 8 and 9 illustrate the elliptical motion described earlier. In both cases the graph is the undirected 6-cycle.

\section{ORIENTABLE FORMATIONS}

We note that if we desire that the formation reorients itself to always "point" in the direction of the motion we need a different kind of feedback law (and indeed a different definition of formation space). The appropriate set up in the 


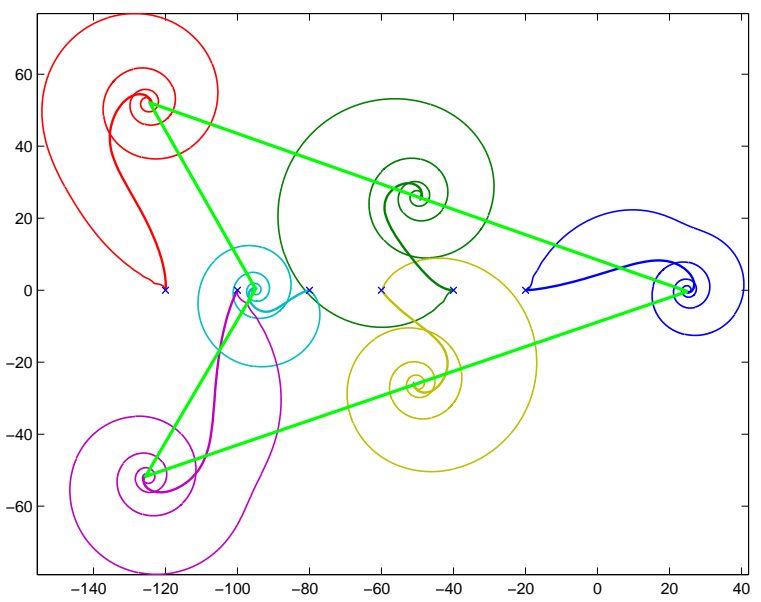

Fig. 7. Rotation with zero initial velocity. $k=1$ (bold), $k=10$ (regular), $f_{1}=-2, f_{2}=-3 . \Gamma$ is undirected cycle.

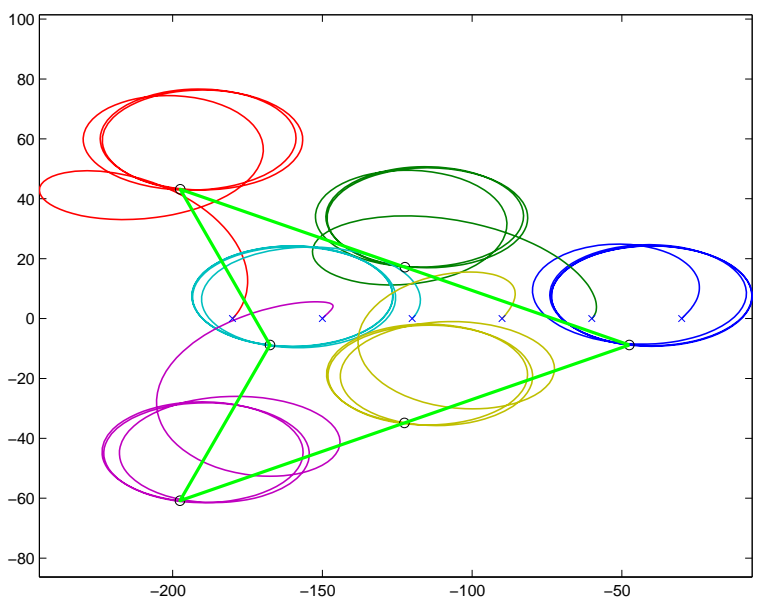

Fig. 8. Elliptical motion, $k=2, f_{1}=-6, f_{2}=-9$.

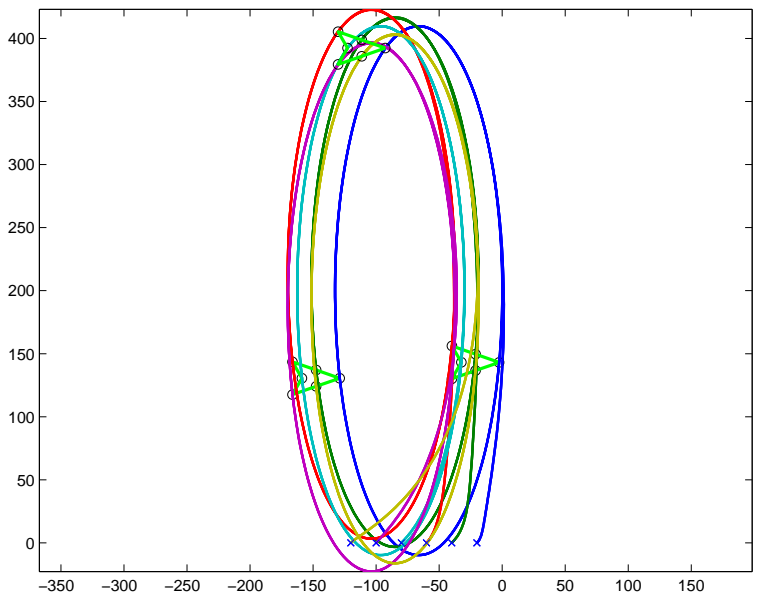

Fig. 9. Elliptical motion, $k \approx 0.3, f_{1}=-2, f_{2}=-3$. case $A_{\text {for }}=0$ is to define the vehicles to be in (oriented) formation $h$ if the state $x(t)=R_{\mathbf{1}_{N} \otimes \alpha} h+\mathbf{1}_{N} \otimes \alpha$ with $\alpha$ satisfying Equation 9 , where $R_{z}$ is (essentially) a rotation matrix that turns a vector into the direction specified by the vector $z$ (this requires $z \neq 0$ ), properly applied one vehicle at a time. It was shown in [16] that under these conditions and using the feedback law $F L\left(z-R_{z} h\right)$ the vehicles converge to formation provided the (negative) gains are large enough and the speed is large enough. Figure 10 illustrates this in case the communication graph is the undirected cycle in six vertices. There a three traces corresponding to three different initial velocities. The formation orients itself in the direction of motion. It is important to note that these new equations are non-linear and thus much harder to analyze.

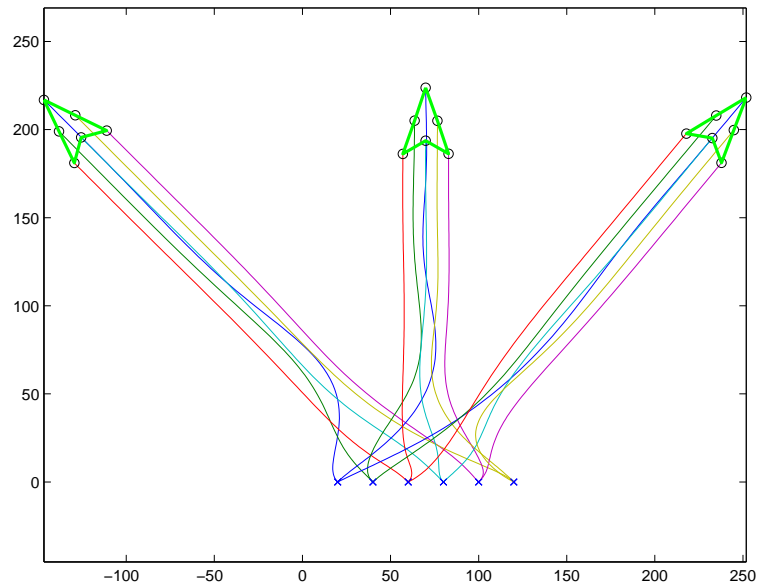

Fig. 10. Reorientation of the formation for three different sets of initial velocities.

We also illustrate the motion in the case when the matrix $A_{\text {for }}$ has nonzero off-diagonal terms (Figures 11 and 12). Notice that, while the reorientation is not exactly in the direction of motion, there appears to be a stable limit cycle (notice the almost superimposed formations on the right of Figure 12). Moreover, it appears that the vehicles are close to formation while they perform this maneuver. This can be used in two different ways. If the goal is to maintain that maneuver but with the formation exactly oriented in the direction of motion one could specify a ghost initial formation that would cancel out the offset. If the goal is to perform a temporary maneuver until straight motion resumes, the model as is maintains the vehicles close enough to formation so that it can be restored later.

The new feedback law $F L\left(z-R_{z} h\right)$ depends on using the vehicles own velocities to compute $R_{z} h$ (as opposed to their velocities relative to their neighbors). However, computing the velocity depends on local information. More precisely, the vehicles can compute their velocity without knowing the global origin of coordinates, but just a translate of the axis.

\section{CONCLUDING REMARKS}

We have extended earlier results guaranteeing convergence to formation for various vehicle dynamics. We established 


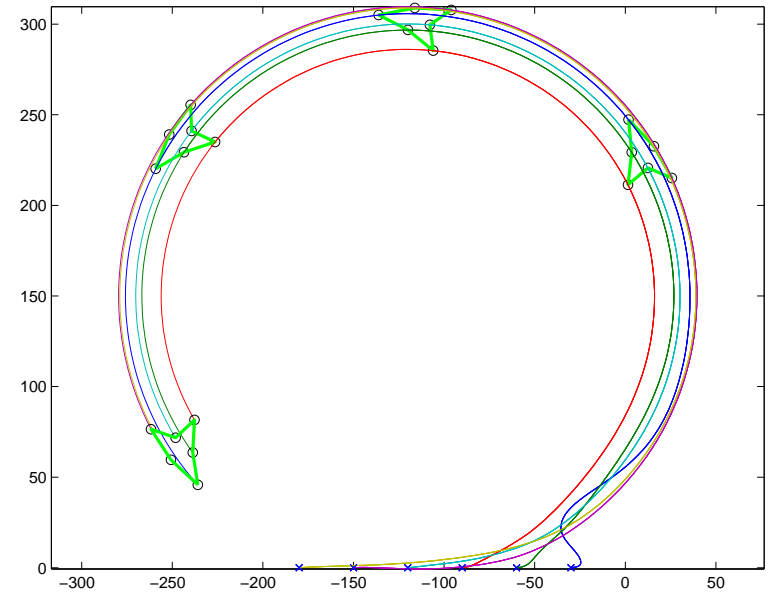

Fig. 11. Rotation with $k=0.1, f_{1}=-2, f_{2}=-3 . \Gamma$ is the complete graph.

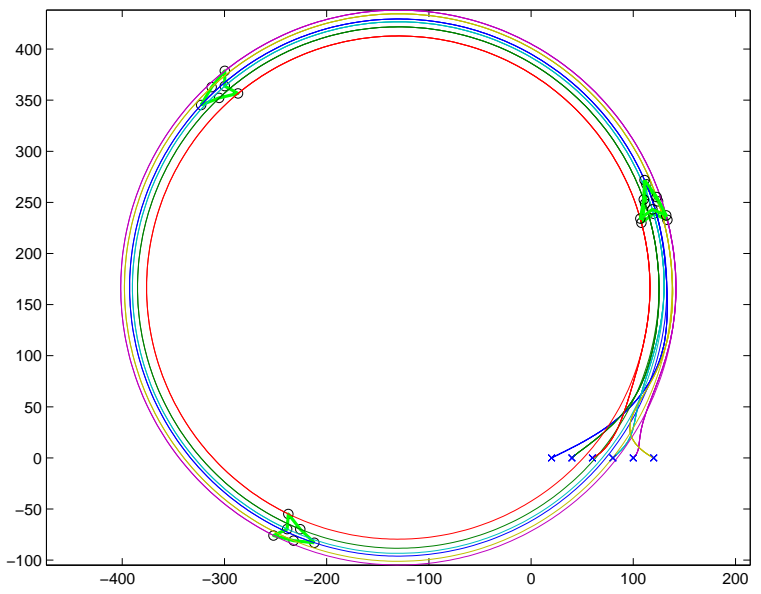

Fig. 12. Rotation with $k=0.5, f_{1}=-2, f_{2}=-3 . \Gamma$ is the undirected 6-cycle.

that, in the case that the eigenvalues of the Laplacian are real, circular and elliptical motions are stable regardless of the radius (and eccentricity) if and only if the feedback gains are negative. This covers all cases of bidirectional communications. For the case of complex eigenvalues we provide necessary and sufficient conditions on the values of $k$ and the gains that guarantee convergence to formation. We also discussed some stable non-linear feedback laws that achieve reorientation of the formation in the direction of motion.

\section{REFERENCES}

[1] J. A. Fax, "Optimal and cooperative control of vehicle formations," Ph.D. dissertation, California Institute of Technology, 2001.

[2] J. Fax and R. Murray, "Information flow and cooperative control of vehicle formations," IEEE Transactions on Automatic Control, vol. 49 , no. 9, pp. 1465-1476, 2003.

[3] S. Glavaski, M. Chavez, R. Day, L. Gomez-Ramos, P. Nag, A. Williams, and W. Zhang, "Vehicle networks: Achieving regular formation," IMA Technical Report, 2002.

[4] A. Jadbabaie, J. Lin, and A. Morse, "Coordination of groups of mobile autonomous agents using nearest neighbor rules," IEEE Transactions on Automatic Control, vol. 48, no. 6, pp. 998-1001, June 2003.
[5] G. Lafferriere, J. Caughman, and A. Williams, "Graph theoretic methods in the stability of vehicle formations," ACC2004, pp. 37293724, July 2004.

[6] G. Lafferriere, A. Williams, J. Caughman, and J. J. P. Veerman, "Decentralized control of vehicle formations," Systems and Control Letters, 2005, to appear. http://www.mth.pdx.edu/ gerardo/papers/vehicles.pdf.

[7] J. Lawton, R. Beard, and B. Young, "A decentralized approach to formation maneuvers," IEEE Transactions on Robotics and Automation, vol. 19, no. 6, pp. 933-941, December 2003.

[8] N. Leonard and E. Fiorelli, "Virtual leaders, artificial potentials and coordinated control of groups," Proceedings of IEEE Conference on Decision and Control, pp. 2968-2973, 2001.

[9] N. Leonard and P. Ogren, "Obstacle avoidance in formation," IEEE ICRA, pp. 2492 - 2497, September 2003.

[10] W. Ren and R. Beard, "A decentralized scheme for spacecraft formation flying via the virtual structure approach," AIAA Journal of Guidance, Control and Dynamics, vol. 27, no. 1, pp. 73-82, January 2004.

[11] A. Sparks, "Special issue on control of satellite formations," International Journal of Robust and Nonlinear Control, vol. 12(2-3), 2002.

[12] H. Tanner, A. Jadbabaie, and G. Pappas, "Stable flocking of mobile agents, part I: Fixed topology," in Proc. IEEE Conference on Decision and Control, December 2003, pp. 2010-2015.

[13] — - "Stable flocking of mobile agents, part II: Dynamic topology," in Proc. IEEE Conference on Decision and Control, December 2003, pp. 2016-2021.

[14] _ , "Flocking in fixed and switching networks," Automatica, Submitted July 2003.

[15] A. Williams, S. Glavăski, and T. Samad, "Formations of formations: Hierarchy and stability," ACC2004, pp. 2992-2997, July 2004.

[16] J. J. P. Veerman, G. Lafferriere, J. S. Caughman, and A. Williams, "Flocks and formations," 2004, submitted to J. Stat. Phys. http://www.mth.pdx.edu/ veerman/flocks2.pdf.

[17] W. Ren and R. Beard, "Consensus seeking in multi-agent systems using dynamically changing interaction topologies," IEEE Trans. Automat. Control, To appear.

[18] D. B. West, Introduction to Graph Theory. London: Prentice Hall, 2001

[19] F. R. K. Chung, Spectral Graph Theory, ser. CBMS Regional Conference Series in Mathematics. Providence, RI: American Mathematical Society, 1997.

[20] R. Brualdi and H. Ryser, Combinatorial Matrix Theory, ser. Encyclopedia of Mathematics and Its Applications. Cambridge University Press, 1991, vol. 39.

[21] C. D. Godsil, Algebraic Combinatorics. New York: Chapman and Hall, 1993. 